\title{
Levels of dopamine, 5-hydroxytryptamine and their metabolites in the striatum of rats of various strains receiving a high-calorie diet
}

\section{Sergey Apryatin ${ }^{1}$, Evgenia Efimova ${ }^{2}$, Zoya Fesenko², Antonina Shumakova ${ }^{3}$, and Ivan Gmoshinski ${ }^{3}$}

${ }^{1}$ Institute of Experimental Medicine, ul. Akademika Pavlova, 12, Saint Petersburg, 197376, Russian Federation

2Institute of Translational Biomedicine, Saint Petersburg State University, Universitetskaya nab., 7-9, Saint Petersburg, 199034, Russian Federation ${ }^{3}$ Federal Research Centre of Nutrition and Biotechnology, Ustyinsky proezd, 2/14, Moscow, 109240, Russian Federation

Address correspondence and requests for materials to Ivan Gmoshinski, gmosh@ion.ru

\section{Abstract}

Citation: Apryatin, S., Efimova, E.,

Fesenko, Z., Shumakova, A., and

Gmoshinski, I. 2021. Levels of dopamine,

5-hydroxytryptamine and their metabolites

in the striatum of rats of various

strains receiving a high-calorie diet.

Bio. Comm. 66(4): 326-332. https://doi. org/10.21638/spbu03.2021.405

Author's information: Sergey Apryatin, PhD, Senior Researcher, orcid.org/00000002-6892-4387; Evgenia Efimova, PhD, Senior Researcher, orcid.org/0000-00029641-515X; Zoya Fesenko, Research Assistant, orcid.org/0000-0002-20749081; Antonina Shumakova, PhD, Senior Researcher, orcid.org/0000-0003-1373-4436; Ivan Gmoshinski, Dr. of Sci. in Biology, Leading Researcher, orcid.org/0000-0002 3671-6508

Manuscript Editor: Michael Firsov, Sechenov Institute of Evolutionary Physiology and Biochemistry, Russian Academy of Sciences, Saint Petersburg, Russia

Received: March 24, 2021:

Revised: May 18, 2021

Accepted: May 26, 2021.

Copyright: @ 2021 Apryatin et al. This is an open-access article distributed under the terms of the License Agreement with Saint Petersburg State University, which permits to the authors unrestricted distribution, and self-archiving free of charge.

Funding: The work was supported by a grant from the Russian Science Foundation, No. 17-16-01043, for the behavioral testing of animals and distribution into groups, drawing up of experimental diets, animal feeding and biological material collection from the striatum. Measurement of monoamine levels and genotyping of animals was supported by project ID 51143531 of the St. Petersburg State University, St. Petersburg, Russia.

Ethics statement: The work with animals was carried out in accordance with Directive 2010/63/EU on the protection of animals used for scientific purposes adopted on September 22, 2010. The design of the experiment was approved by the Ethics Committee of the Federal Research Centre of Nutrition and Biotechnology (protocol No. 4 of 04/20/2017).

Competing interests: The authors have declared that no competing interests exist.
The aim of this work was to study the effect of a high fat and carbohydrate diet (HFCD) and quercetin supplementation on the levels of dopamine (DA), serotonin (5-HT) and their metabolites in Wistar, DA transporter knockout (DAT$\mathrm{KO}$ ) and obese Zucker fa/fa rats. Animals received a control diet or HFCD for 62 days. Wistar and Zucker fa/fa rats received quercetin. The contents of DA, 5-HT, norepinephrine (NE), dioxyphenyl acetate (DOPAC), homovanillic acid (HVA) and 5-hydroxyindolyl acetate (5-HIIA) in the striatum were determined by high-performance liquid chromatography (HPLC). DAT-KO homozygotes had lowered DA and increased HVA and DOPAC compared to Wistar rats. HFCD did not affect the content of NE and 5-HT. 5-HT was increased in DAT-KO homozygotes compared with Wistar receiving a control diet. 5-HIIA accumulated in larger amounts in DAT-KO compared to Wistar with the exception of those receiving quercetin with a control diet. Quercetin did not affect the levels of DA, 5-HT and their metabolites.

Keywords: dopamine, 5-hydroxytryptamine, rats, striatum, fat and carbohydrate excess, quercetin

\section{Introduction}

According to modern data, the central role in body energy homeostasis belongs to the system of interacting serotonergic and dopaminergic CNS neurons (Burke and Heisler, 2015; Volkow et al., 2011). Synthesis and exchange of dopamine (DA) and serotonin (5-hydroxytryptamine, 5-HT) occur both in the brain and in peripheral tissues; however, only DA and 5-HT synthetized in the brain are significant for the neuroendocrine regulation of energy metabolism, hunger, satiety and nutritional behavior since these biogenic amines are essentially unable to pass through the blood-brain barrier. The predominant part of the synthesized DA and $5-\mathrm{HT}$ is concentrated in the midbrain regions, including the striatum and substantia nigra, where important centers associated with feeding behavior are located. Previously, it was shown that the response of behavioral reactions and biochemical indexes to the consumption of a diet with an excess of simple carbohydrates and fat differs in the dopamine reverse transporter gene (DAT)knock-out rats (DAT-KO) and wild-type animals (Apryatin et al., 2019). However, the question of how these differences are related to the metabolism of biogenic amines in the brain remains unclear. In the development of modern methods of diet therapy for obesity, metabolic syndrome and related conditions, much attention is given to biologically active polyphenolic substances, which can 
actively influence energy homeostasis and eating behavior. According to a number of experimental studies (Panchal et al., 2012) and clinical observations (Edwards et al., 2007), quercetin (Q; 3,3'4,5,7-pentahydroxyflavone) can alleviate the symptoms of metabolic syndrome caused by excess consumption of easily digestible carbohydrates. In this regard, Q attracts special attention as a promising remedy for dietetic treatment of metabolic syndrome and obesity. The mechanisms of the effect of $\mathrm{Q}$ and other polyphenols on food energy balance in the body are not completely understood. In particular, the ability of this substance to influence the brain levels of DA, 5-HT and its metabolites, which determine feeding behavior, after oral intake, has not been studied.

The aim of this work was to study the effect of consumption of a hypercaloric diet with a relative excess of fat and carbohydrates and $\mathrm{Q}$ supplementation on the levels of DA, 5-HT and their main metabolites in the whole striatum using 3 different strains of rats: "wildtype" outbred Wistar rats, DAT-KO rats (homozygotes and heterozygotes) and hereditarily obese Zucker fa/fa rats with impaired leptin reception.

\section{Materials and methods}

\section{Animals, treatments and sample extraction}

\section{STUDY DESIGN}

Studies were performed on 31 male rats (10-12 weeks old) of the DAT-KO strain (DAT-/- homozygotes and $\mathrm{DAT}+/$ - heterozygotes) obtained from the vivarium of the Institute of Translational Biomedicine, St. Petersburg State University (St. Petersburg), as well as 32 males of outbred Wistar rats $(\mathrm{DAT}+/+)$ of the same age received from the nursery of the Stolbovaya branch of the Federal State Budgetary Institution Scientific Center for Biomedical Technologies of FMBA of Russia, and 24 male rats (age 8-10 weeks) of the Zucker (fa/fa) strain (denoted further as "Zucker") obtained from Charles River Nursery, Italy. Every effort was made by us to minimize the number of animals used and to reduce their suffering.

The animals were divided into a total of 12 groups: two (groups 1 and 2) with DAT-/- rats, two (groups 3 and 4) with DAT+/- rats, four (groups 5 to 8) with Wistar rats and 4 (groups 9 to 12) with Zucker rats. The numbers of animals in these groups were 4, 6, 12, 9, 8, 8, 8, 8, $6,6,6$, and 6 , respectively. Within 62 days, animals of the $1^{\text {st }}, 3^{\text {rd }}, 5^{\text {th }}$ and $9^{\text {th }}$ groups (control) received a balanced semisynthetic diet according to AIN93M; the $2^{\text {nd }}, 4^{\text {th }}, 7^{\text {th }}$ and $11^{\text {th }}$ groups received a high-carbohydrate-high-fat diet (HFCD) with $30 \%$ fat by weight of solids and water replaced with a solution of $20 \%$ fructose; the $6^{\text {th }}$ and $10^{\text {th }}$ groups received a control diet with the addition of $\mathrm{Q}$; and the $8^{\text {th }}$ and $12^{\text {th }}$ groups received the HFCD with the addition of Q. Q was added to the rats' diet in an amount from 0.4 to $1.3 \mathrm{~g} / \mathrm{kg}$ of feed (by weight of dry matter) in various groups of animals, which corresponded to a consumed dose of $50 \mathrm{mg} / \mathrm{kg}$ body weight. The amount of quercetin added to the diet was adjusted once every two days according to body weight and mean diet consumption in the previous two days for two rats. Q was purchased from Sigma-Aldrich (product number q4951, $\geq 95 \%$ purity (HPLC)). The dose of Q fed to animals was 100 times greater compared to adequate daily intake in humans $(0.5 \mathrm{mg} / \mathrm{kg} \mathrm{bw})$, which is explained by the need to identify the metabolic effect of this substance, given the greater intensity of metabolic processes in rodents than humans and the relatively short term of the subacute experiment. This dose of quercetin was close to that used in a number of studies on rodents (i.e., Panchal, 2012).

Animals were given unlimited free access to the diet and fluids. Rats were placed in pairs in polycarbonate cages at a 12/12-hour light/dark exposure and an air temperature of $22 \pm 1^{\circ} \mathrm{C}$. The amount of feed and fluid consumed was recorded daily. Body weight was determined weekly on electronic balances with an accuracy of $\pm 1 \mathrm{~g}$.

\section{DAT-KO GENOTYPING}

Before the distribution of DAT-KO rats into experimental groups, they were classified by the allelic type of the DAT gene. Preliminary classification was carried out according to the trajectory of the animals in the open field installation using equipment manufactured by the Panlab Harvard Apparatus Company (Spain). The results of this classification detailed earlier (Apryatin et al., 2019) were confirmed at the end of the experiment by analysis of the allelic type of the DAT gene by real-time PCR followed by restriction analysis with the BtsIMutI restriction enzyme according to Leo et al. (2018).

\section{SAMPLING}

The animals were removed from the experiment through deep anesthesia by inhalation of diethyl ester (analytical grade, produced by JSC "Medkhimprom", Russia, Moscow region, the city of Zheleznodorozhny) followed by decapitation. The skull box was quickly opened, and after removal of the cerebral cortex, the striatum was isolated, which was immediately cooled to $-80^{\circ} \mathrm{C}$ and stored at this temperature until analysis.

\section{HPLC measurements}

HPLC measurements of tissue DA, 5-HT, norepinephrine (NE) and their metabolites 3,4-dihydroxyphenylacetic acid (DOPAC), homovanillic acid (HVA) and 5-hydroxyindoleacetic acid (5-HIAA) were carried out 
(a)

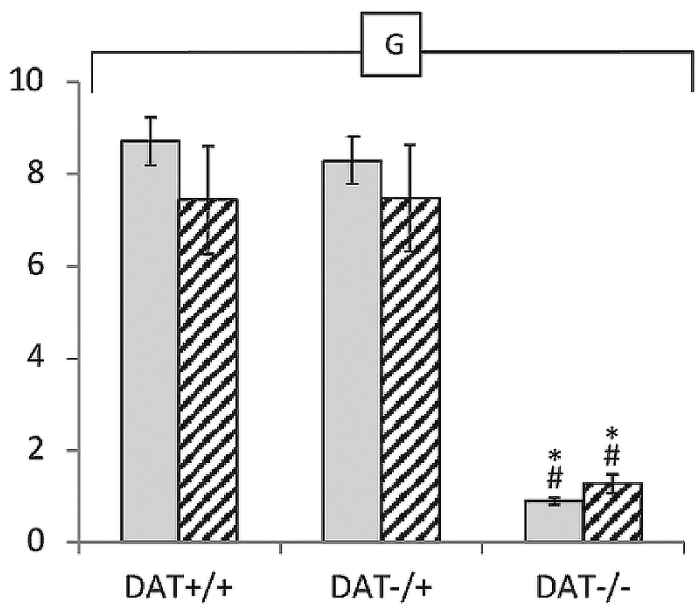

(c)

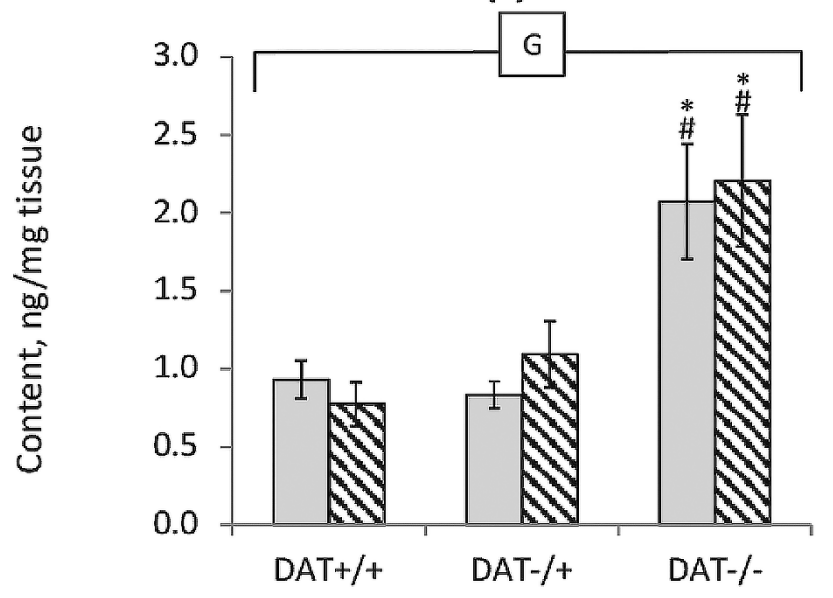

(e)

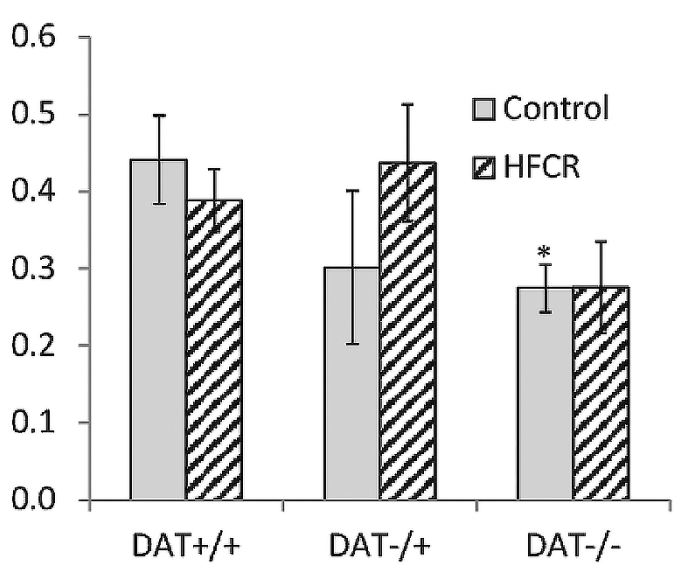

Control (b)

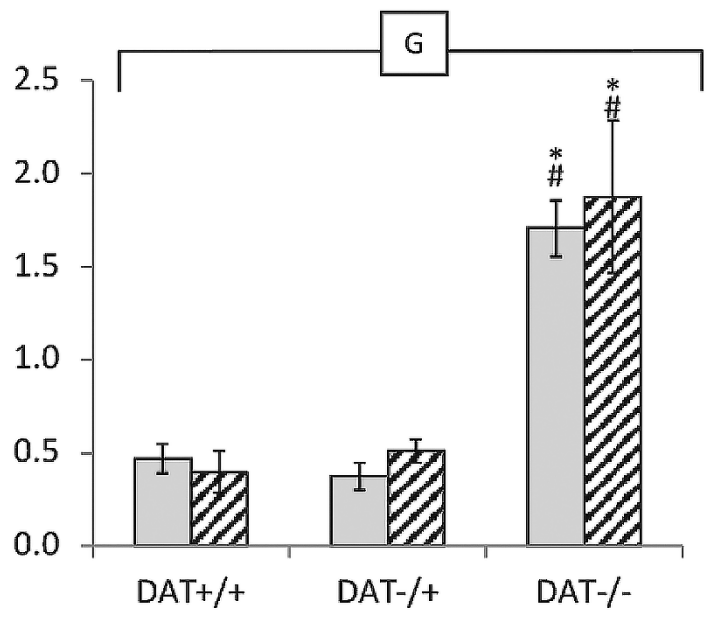

(d)

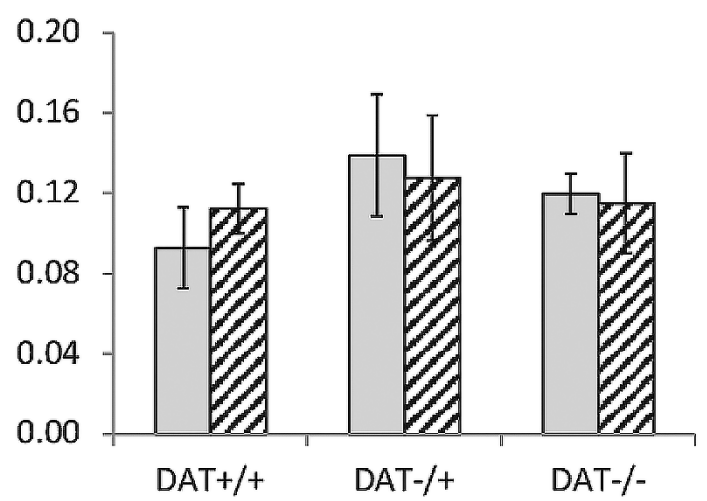

(f)

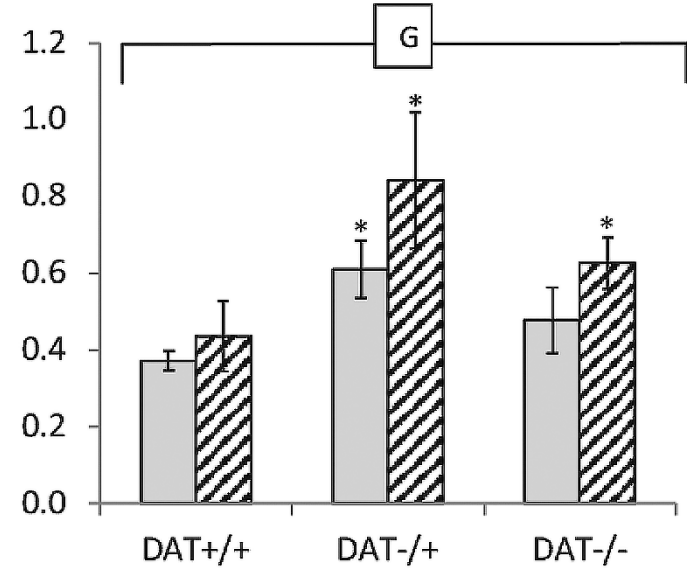

HFCD

Fig. 1. The content of biogenic amines and their metabolites in the striatum of rats with knockout of the dopamine transporter gene (DAT-/-), heterozygotes (DAT+/-) and "wild"-type (DAT+/+; Wistar strain), which received a balanced control and high-carbohydrate, high-fat diet (HFCD). a) dopamine (DA), b) homovanillic acid (HVA), c) dioxyphenyl acetate (DOPAC), d) norepinephrine (NE), e) 5-hydroxytryptamine (5-HT), f) 5-hydroxyindolyl acetate (5-HIIA). * - the difference with DAT+/+ is significant; \# - the difference with DAT+/- is significant for the corresponding diet, $p<0.05$, Mann-Whitney $\cup$ test. Horizontal bracket - the distribution is not uniform, $p<0.05$, two-way analysis of variance, by the factor " $\mathrm{G}$ " (genotype, strain) for the covered range of groups. 
as described previously (Belov et al., 2020). The samples for analysis were homogenized in $0.1 \mathrm{M} \mathrm{HClO}_{4}$, centrifuged $\left(10 \mathrm{~min},+4^{\circ} \mathrm{C} ; 14,000 \mathrm{~g}\right)$ and filtered using centrifuge filter units (PVDF membrane, pore size $0.22 \mu \mathrm{m}$, Millipore). Measurements of DA, 5-HT, NE and their metabolites in the tissue samples were performed using HPLC with electrochemical detection (Eicom, HTEC500, Japan) with a carbon electrode WE-3G (Eicom, Japan) using a $+650 \mathrm{mV}$ applied potential. The system was equipped with a CA-50DS reverse-phase column $\left(150^{\star} 2.1 \mathrm{~mm}\right.$, Eicom, Japan) at a flow rate of $200 \mu \mathrm{l} / \mathrm{min}$. The mobile phase contained $100 \mathrm{mM}$ sodium-phosphate buffer, $0.17 \mathrm{mM}$ EDTA, $1.8 \mathrm{mM}$ octanesulfonic acid sodium salt and $18 \%$ (vol/vol) methanol, $\mathrm{pH}$ 4.5. All peaks obtained were normalized to the internal standard 3,4-dihydroxybenzylamine, and final values for monoamines were expressed as $\mathrm{ng} / \mathrm{mg}$ wet tissue weight.

\section{Statistics}

Statistical data processing was performed using the SPSS 20.0 software package. The homogeneity distribution hypothesis of the compared groups was tested using twoand three-factor ANOVA of variance for DAT-KO and Wistar plus Zucker rats, respectively. Post hoc analysis was performed using a nonparametric Mann-Whitney rank test.

\section{Results}

\section{Levels of biogenic amines and their metabolites in the striatum in a model of rats with impaired dopamine transport}

As follows from the data presented in Fig. 1, homozygous DAT-/- rats were characterized by significantly lower DA levels (Fig. 1a) in the striatum together with almost the same increase in its metabolites HVA (Fig. 1b) and DOPAC (Fig. 1c) when compared with the heterozygous DAT+/- and with wild-type rats (DAT+/+, Wistar strain). According to two-way analysis of variance, the effect of the animal's genotype on these indicators was statistically significant (ANOVA, $p<0.05$ by the "strain" factor). This was confirmed by a significant difference in these parameters between DAT-/- animals and DAT+/- and DAT+/+ receiving the corresponding diets by pairwise comparison $(p<0.05$, Mann-Whitney test). At the same time, the intake of HFCD did not significantly affect the levels of DA or any of its metabolites in rats of all three genotypes. The NE content (Fig. 1d) in the striatum of all rat genotypes receiving both diets did not differ significantly.

The intake of HFCD did not affect the content of 5-HT (Fig. 1e) in the rat striatum of any of the three genotypes. Factor analysis showed the absence of reli- able effects on this indicator from both the strain (genotype) and the diet. However, when comparing DAT-/animals with DAT $+/+$, there was a significant decrease in the accumulation of this biogenic amine in the former (for animals receiving a control diet, the difference was statistically significant, $p<0.05$, Mann-Whitney U test). The metabolite of 5-hydroxytryptamine, namely, 5-HIIA (Fig. 1f), accumulated in significantly large quantities in both the DAT+/- and DAT+/- striatum compared to the DAT $+/+$ striatum (ANOVA, $p<0.05$ according to the "strain" factor). At the same time, the type of diet used did not have a significant effect on this indicator.

\section{Levels of biogenic amines and their metabolites in the striatum in a model of rats with impaired reception of leptin}

The data presented in Figure 2 show that neither the strain of animals nor the intake of HFCD and Q supplementation significantly affected the levels of DA (Fig. 2a), its metabolites (Figs. 2b-2d), or 5-HT (Fig. 2e) in the striatum. The content of 5-HIIA (Fig. 2f) was significantly increased in Zucker rats of all groups compared with the corresponding groups of Wistar rats at all dietary regimes, except for $\mathrm{Q}$ supplementation of the control diet (ANOVA, $p<0.05$ according to the "strain" factor).

\section{Discussion}

When analyzing the results obtained, it should be borne in mind that Wistar rats are considered the parental strain used in DAT-KO breeding and are characterized by the greatest similarity in allelic composition of genes, with the exception of DAT. Under these conditions, as shown in Figure 1, the only factor affecting the levels of DA and all its metabolites, with the exception of NE, was the genotype (allelic type of DAT). Namely, there was a significant and multi-fold decrease in the level of DA and, correspondingly, an increase in the content of DOPAC and HVA in DAT-/- homozygotes in comparison with both heterozygotes and Wistar rats. These data coincided with the results of Leo et al. (2018), who demonstrated a marked decrease in the total striatal DA content together with an increase in DOPAC and HVA.

This result is in apparent contradiction with the violation of DA transport in DAT-KO, leading to the accumulation of this compound in the synaptic cleft. In fact, it should be considered that, in normal animals, DA, when captured by presynaptic neurons, mainly accumulates in vesicles for subsequent reutilization or is metabolized (oxidized) under the influence of monooxygenase with the formation of NE. In DAT-KO, DA that accumulates in the synaptic cleft is not reabsorbed but diffuses into the intercellular space, from which it 
(a)

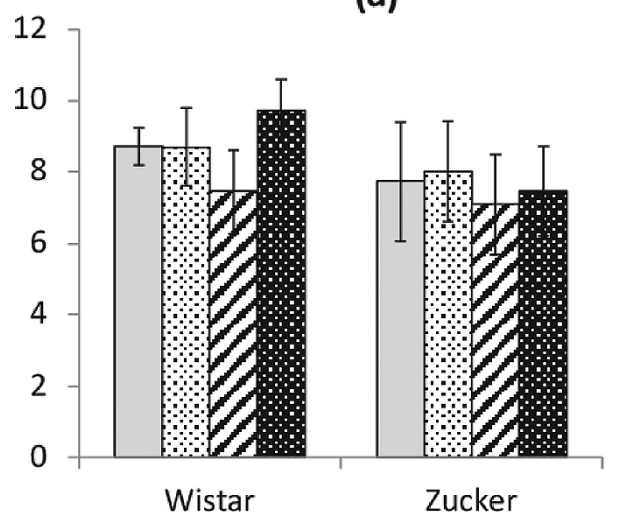

(c)

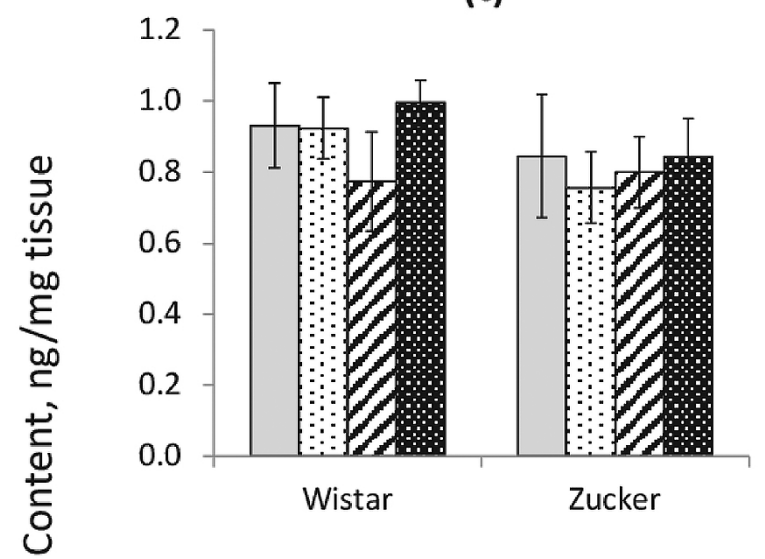

(e)

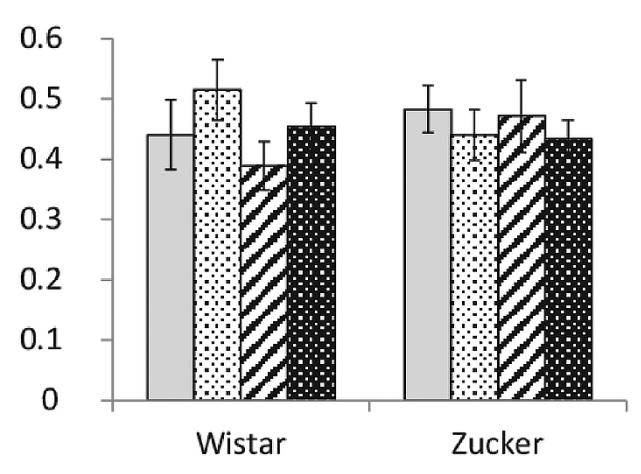

(b)

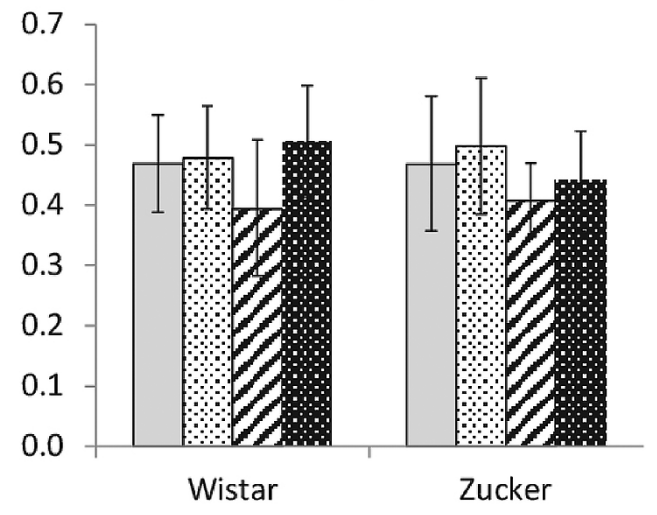

(d)

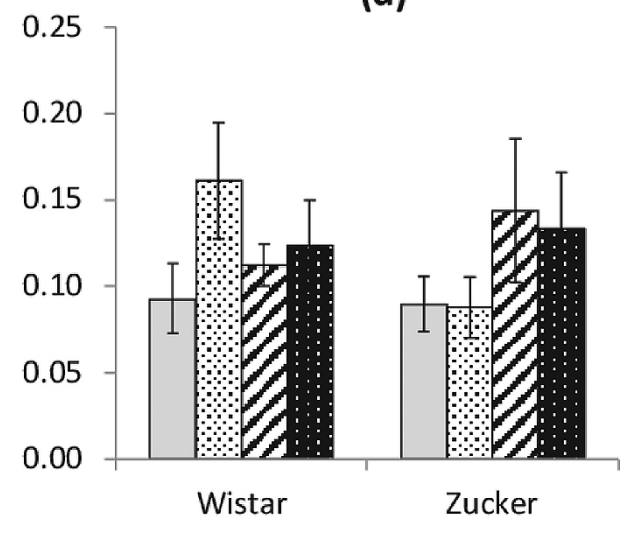

(f)

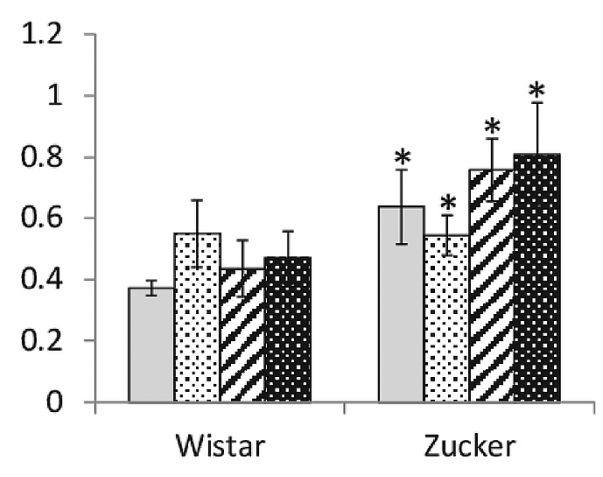

\section{$\square$ Control $\quad$ Control+Q $\square \mathrm{HFCD} \quad$ HFCD+Q}

Fig. 2. The content of biogenic amines and their metabolites in the striatum of rats with impaired (Zucker fa/fa) and normal (Wistar) reception of leptin that received a balanced control diet or HFCD and quercetin supplementation for 60 days. a) dopamine (DA), b) homovanillic acid (HVA), c) dioxyphenyl acetate (DOPAC), d) norepinephrine (NE), e) 5-hydroxytryptamine (5-HT), f) 5-hydroxyindolyl acetate (5-HIIA) * — the difference with Wistar rats is significant for the corresponding diet, $p<0.05$, Mann-Whitney $\mathrm{U}$ test. Horizontal bracket - the distribution is not uniform, $p<0.05$, two-way analysis of variance, by the factor " $G$ " (genotype, strain) for the covered range of groups. 
can be captured by astroglial cells (Jennings and Rusakov, 2016) expressing biogenic amine transporters that are not affected by DAT knockout. In these cells, DA is subsequently metabolized by monoamine oxidase and aldehyde dehydrogenase to form increased amounts of DOPAC and HVA.

In the conducted experiments, the opposite effect of DAT gene knockout on 5-HT and its metabolite 5-HIIA levels was revealed, which presumably indicates the interaction between the metabolic pathways of DA and 5-HT in the striatum. A possible explanation of such effects is that the 5-HT transporter (SERT) in presynaptic neurons, which carries out its reabsorption from the synaptic cleft, has a certain degree of cross-specificity for DA (Larsen et al., 2011). Under conditions of normal concentrations of DA, this interaction is apparently insignificant; however, when DA accumulation in the synaptic cleft is sharply increased in DAT-KO, DA probably begins to competitively inhibit 5-HT reabsorption. The consequence of this is the inhibition of 5-HT reabsorption by neurons, its entry into the extracellular space and capture by glial cells, followed by metabolism along the aforementioned common pathway with DA.

The elevated 5-HIIA level in Zucker rats compared to Wistar rats indicates a greater degree of 5-HT catabolism in these animals compared to Wistar rats. According to the literature, the formation of 5-HIIA in the brains of rats is controlled by the insular apparatus and accelerates with an increase in insulin levels (Svec et al., 2004). Indeed, Zucker rats are phenotypically characterized by an increased level of insulin (King and Austin, 2017). Another reason for the observed effect on the striatum 5-HIIA content may be the differences in leptin levels. As follows from previously obtained data (Mzhelskaya et al., 2020) in Zucker rats, leptin levels in blood plasma were increased 20 times compared with Wistar rats when consuming a control diet and 50-300 times compared to those who received HFCD. At the same time, Q supplementation did not significantly affect leptin levels. Taken together, this indicates the ability of additional amounts of leptin entering the brain to potentiate 5-HT metabolism, which is consistent with the antagonism of these neurotransmitters known from the literature (Yadav et al., 2011).

The results obtained showed that HFCD had no significant effect on the levels of DA, 5-HT and their main metabolites in normal rats or rats with impaired DA transport (DAT-KO) and leptin reception (Zucker). The available data on the effect of excess caloric intake on DA levels in different parts of the central nervous system are contradictory (Rada et al., 2005, 2010; Geiger et al., 2009). In particular, there are no reports on the effects of long-term intake of excess carbohydrates and fat on DA metabolite levels. According to Wu et al. (2017) the hypothesis about the role of 5-HT transport in the development of obesity has not been supported.
A number of works have reported that $\mathrm{Q}$ is capable of exerting a significant influence on the processes of carbohydrate-energy and fat metabolism in peripheral organs in experimental models of rats and mice (Panchal et al., 2012) and is also able to modulate their behavioral reactions (Mzhelskaya et al., 2020). To a certain extent, the potentiation of $\mathrm{Q}$ action against the background of HFCD feeding would be due to the known effect of increasing the bioavailability of Q with an excess of fat in the diet (Guo et al., 2013). However, in previous work (Mzhelskaya et al., 2020), we showed that the influence of HFCD on the size and even the direction of the effect of quercetin on behavioral responses differs in rats with normal and impaired reception of leptin. Therefore, leptin signaling pathways contribute to the development of the body's response to $\mathrm{Q}$, which should be taken into account in assessing the clinical efficiency of $Q$ intake.

The capability of $\mathrm{Q}$ and its derivatives to affect the metabolism of neurotransmitter amines in the brain has not been studied enough. This possibility is obviously limited by the degree of penetration of these substances through the blood-brain barrier. According to the available data (Costa et al., 2016), only very small nanomolar and picomolar amounts of $\mathrm{Q}$ are found in the brains of rats and pigs after oral administration. As shown by the results of this study, no significant effect of quercetin on the exchange of DA, 5-HT and their metabolites in the striatum of the rat brain was found. The permeability of the brain-blood barrier to $\mathrm{Q}$ and its derivatives in animals of various strains in connection with their behavioral reactions and the exchange of neurotransmitters deserves further research.

\section{Conclusion}

The results obtained in this study indicate the absence of brain DA and 5-HT exchange roles in the effects of a diet with a high specific calorie content on biochemical and behavioral indexes in DAT-KO and Wistar rats. The data obtained also do not support the hypothesis of the direct role of central DA and 5-HT in the effects of Q supplementation in Wistar and Zucker rats noted previously. The capability of $\mathrm{Q}$ and its metabolites to penetrate into the brain in relation to the metabolic and behavioral effects of these compounds in animals of different strains deserves further research.

\section{References}

Apryatin, S. A., Shipelin, V. A., Trusov, N. V., Mzhelskaya, K. V., Evstratova, V. S., Kirbaeva, N. V., Soto, J. S., Fesenko, Z. S., Gainetdinov, R. R., and Gmoshinski, I.V. 2019. Comparative analysis of the influence of a high-fat/highcarbohydrate diet on the level of anxiety and neuromotor and cognitive functions in Wistar and DAT-KO rats. Physiological Reports 7:e13987. https://doi.org/10.14814/ phy2.13987 
Belov, D. R., Efimova, E. V., Fesenko, Z. S., Antonova, K. A., Kolodyazhny, S. F., Lakstygal, A. M., and Gainetdinov, R. R. 2020. Putative trace-amine associated receptor 5 (TAAR5) agonist a-NETA increases electrocorticogram gamma-rhythm in freely moving rats. Cellular and Molecular Neurobiology 40:203-213. https://doi.org/10.1007/ s10571-019-00716-1

Burke, L. K. and Heisler, L. K. 2015. 5-hydroxytryptamine medications for the treatment of obesity. Journal of Neuroendocrinology 27:389-398. https://doi.org/10.1111/ jne. 12287

Costa, L. G., Garrick, J. M., Roquè, P. J., and Pellacani, C. 2016. Mechanisms of neuroprotection by quercetin: counteracting oxidative stress and more. Oxidative Medicine and Cellular Longevity 2016:2986796. https://doi. org/10.1155/2016/2986796

Edwards, R.L., Lyon, T., Litwin, S.E., Rabovsky, A., Symons, J. D., and Jalili, T. 2007. Quercetin reduces blood pressure in hypertensive subjects. Journal of Nutrition 137:2405-2411. https://doi.org/10.1093/jn/137.11.2405

Geiger, B. M., Haburcak, M., Avena, N. M., Moyer, M. C., Hoebel, B. G., and Pothos, E. N. 2009. Deficits of mesolimbic dopamine neurotransmission in rat dietary obesity. Neuroscience 159:1193-1199. https://doi.org/10.1016/j. neuroscience.2009.02.007

Guo, Y., Mah, E., Davis, C. G., Jalili, T., Ferruzzi, G., Chu, O. K., and Bruno, R. S. 2013. Dietary fat increases quercetin bioavailability in overweight adults. Molecular Nutrition \& Food Research 57:896-905. https://doi.org/10.1002/ mnfr.201200619

Jennings, A. and Rusakov, D. A. 2016. Do astrocytes respond to dopamine? Opera Medica et Physiologica 2:34-43. https://doi.org/10.20388/OMP2016.001.0017

King, A. and Austin, A. 2017. Animal models of type 1 and type 2 diabetes mellitus; pp. 245-265 in Conn P. M. (ed.), Animal models for the study of human disease (Second Edition). Academic Press. https://doi.org/10.1016/B9780-12-809468-6.00010-3

Larsen, M. B., Sonders, M. S., Mortensen, O. V., Larson, G. A., Zahniser, N. R., and Amara, S. G. 2011. Dopamine transport by the serotonin transporter: a mechanistically distinct mode of substrate translocation. Journal of Neuroscience. 17:6605-6615. https://doi.org/10.1523/JNEUROSCl.0576-11.2011

Leo, D., Sukhanov, I., Zoratto, F., Illiano, P., Caffino, L., Sanna, F., Messa, G., Emanuele, M., Esposito, A., Dorofeikova, M., Budygin, E. A., Mus, L., Efimova, E. V., Niello, M.,
Espinoza, S., Sotnikova, T. D., Hoener, M. C., Laviola, G., Fumagalli, F., Adriani, W., and Gainetdinov, R. R. 2018. Pronounced hyperactivity, cognitive dysfunctions, and BDNF dysregulation in dopamine transporter knock-out rats. Journal of Neuroscience 38(8):1959-1972. https:// doi.org/10.1523/JNEUROSCI.1931-17.2018

Mzhelskaya, K. V., Shipelin, V.A., Shumakova, A.A., Musaeva, A. D., Soto, J.S., Riger, N. A., Apryatin, S. A., and Gmoshinski, I. V. 2020. Effects of quercetin on the neuromotor function and behavioral responses of Wistar and Zucker rats fed a high-fat and high-carbohydrate diet. Behavioural Brain Research. 378:112270. https://doi. org/10.1016/j.bbr.2019.112270

Panchal, S. K., Poudyal, H., and Brown, L. 2012. Quercetin ameliorates cardiovascular, hepatic, and metabolic changes in diet-induced metabolic syndrome in rats. Journal of Nutrition 142:1026-1032. https://doi.org/10.3945/ jn.111.157263

Rada, P., Avena, N. M., and Hoebel, B. G. 2005. Daily bingeing on sugar repeatedly releases dopamine in the accumbens shell. Neuroscience 134:737-744. https://doi. org/10.1016/j.neuroscience.2005.04.043

Rada, P., Bocarsly, M. E., Barson, J. R., Hoebel, B. G., and Leibowitz, S. F. 2010. Reduced accumbens dopamine in Sprague-Dawley rats prone to overeating a fat-rich diet. Physiology \& Behavior 101:394-400. https://doi. org/10.1016/j.physbeh.2010.07.005

Svec, F., Robinson, P., Michel, L., Bradley, J., Corll, C., and Porter, J. R. 2004. Caloric intake and hypothalamic neurotransmitters in Zucker rats made acutely diabetic with streptozocin. Nutritional Neuroscience 7:317-324. https://doi.org/10.1080/10284150400020508

Volkow, N. D., Wang, G. J., and Baler, R. D. 2011. Reward, dopamine and the control of food intake: implications for obesity. Trends in Cognitive Sciences 15:37-46. https:// doi.org/10.1016/j.tics.2010.11.001

Wu, C.-H., Chang, C.-S., Yang, K. Y., Shen, L.-H., and Yao, W.-J. 2017. Comparison of brain serotonin transporter using [123I]-ADAM between obese and non-obese young adults without an eating disorder. PLOS One 12:e0170886. https://doi.org/10.1371/journal.pone.0170886

Yadav, V. K., Oury, F., Tanaka, K., Thoma, T., Wang, Y., Cremers, S., Hen, R., Krust, A., Chambon, P., and Karsenty, G. 2011. Leptin-dependent serotonin control of appetite: temporal specificity, transcriptional regulation, and therapeutic implications. Journal of Experimental Medicine 208:41-52. https://doi.org/10.1084/jem.20101940 